\title{
UNA VERSIÓN DE LA FÁBULA DE LA LECHERA EN PLAUTO
}

\author{
M. ${ }^{z}$ Victoria Fernández-Savater Martin
}

UNED

\section{RESUMEN}

Entre las numerosas versiones del cuento que conocemos como «fábula de la lechera", que no es sino una recreación del motivo "castillos en el aire", destaca por su originalidad y singularidad la que encontramos en el comediógrafo latino Plauto en su obra Rudens. El análisis detenido del texto nos permitirá observar la adaptación de un relato, sólidamente fijado, a la escena, los motivos que determinan, propician y componen esta nueva versión de la fábula, y la función esencial que dentro del argumento desempeña.

Cuando hablamos de la «fábula de la lechera» no suele ser necesario añadir nada más; todos sabemos a qué cuento nos estamos refiriendo y, sin duda, la mayoría de nosotros podría contarlo con más o menos exactitud. La versión que conocemos, la que contaríamos, es la que llevó a cabo el fabulista Samaniego tomando como ejemplo aquella que elaboró magníficamente La Fontaine, a quien pudieron llegar las versiones que aparecen en el Dialogus creaturarum optime moralizatus atribuido a Nicolás de Pérgamo y en los Sermones de Jacques de Vitry ', ya que, según parece, es precisamente en estas versio-

1 En Vitry la mujer es una vieja avarienta, sin embargo, en Nicolás de Pérgarno se trata de una jovencita a quien su ama ha enviado a la ciudad para vender el cántaro de leche. Desconocemos en qué momento del camino surgió este cambio en la fábula. 
nes en las que por vez primera «la lechera» es una lechera; es decir, la primera vez que quien hace «castillos en el aire» ${ }^{2}$ finalmente truncados es una mujer con un cántaro de leche, y no un brhaman con su bote de harina, un vendedor de cristalería con su frágil carga, o un religioso y un ermitaño con su jarra de miel. Todos estos personajes que hicieron «castillos en el aire» antes, según se cree, que nuestra lechera, pertenecen a las colecciones de cuentos orientales.

El apasionante y complejo estudio del camino seguido por las diferentes versiones de esta fábula, desde el Panchatantra hasta Samaniego pasando por diferentes literaturas, fue llevado a cabo de forma brillante en primer lugar por F. MAX MÜLLER ${ }^{3}$ cuya labor han continuado investigadores de gran relevancia, que llegan incluso a estudiar versiones en nuestra literatura más reciente ${ }^{4}$; no considero oportuno repetir lo que otros han desarrollado de forma tan acertada, tan sólo me gustaría resaltar el posible origen oriental de la fábula primera en la que se dan los rasgos principales del cuento que luego se irán alterando en las diferentes versiones: un personaje pobre que, a partir de una pequeña posesión, sueña despierto cómo se irá enriqueciendo y convirtiendo en alguien diferente, hasta que, generalmente por un error propio, el objeto en el que se ponen las esperanzas se pierde.

Una de estas versiones, totalmente diferente a todas las demás que conocemos es la que encontramos inserta en la comedia Rudens ${ }^{5}$ de Plauto ${ }^{6}$, el más popular de los comediógrafos latinos. La trama de la historia es, más o menos, la que sigue:

${ }^{2}$ El folklorista Tномрsом, S., en su obra Motif-Index of Folk-Literature, Copenhague-Blomington-Indiana 1955-58, vols. 6, clasifica todas estas versiones que van cambiando algún rasgo del cuento, en el motivo general «castillos en el aire» J2061.

${ }^{3}$ MAX Müller, F., (1823-1900) en su estudio «La emigración de las fábulas», conferencia pronunciada en la Institución Real de Londres en 1870, y luego incluido en la obra que yo manejo en una traducción española hecha a partir del inglés (Comparative Mythology): Mitología Comparada, Barcelona 1988.

4 Mille y Jiménez, J., (1924) «La fábula de la lechera al través de las diversas literaturas», Nosotros 48, 203-225; y, sobre todo, el esclarecedor estudio de Fradejas Lebrero, J. (1978) «Algunas versiones más de la fábula de la lechera», Cuadernos para la Investigación de la Literatura Hispánica 1,21-30. Fue precisamente este último el que me hizo recordar el pasaje de Plauto que aquí examino y que siempre me había llamado la atención de manera especial.

s El texto latino que empleo pertenece a la edición de A. Ernout en Les Belles lettres, París 1962.

- En otras comedias de Plauto aparecen alusiones a fábulas, como por ejemplo en Pseudolus a «El carnero guardado por el lobo» ( $v v 139 \mathrm{ss}$.), o aplicaciones de estas como en Aulularia en donde «El buey y el asno» se emplea para representar simbólicamente al rico y al pobre (vv.226 ss.); en ambos casos se trata de versiones conocidas. 
Démones, a quien hace años raptaron a su hija Palestra, vive en Cirene, y no sabe que en la misma ciudad vive el lenón Lábrax que la compró ${ }^{7}$; Pleusidipo, joven enamorado de Palestra, ha pagado la mitad del precio que acordó con el lenón por la compra de la joven, pero éste, rompiendo el trato, ha huido en barco, con nocturnidad y alevosía ${ }^{8}$, llevándose a Palestra; naufragan y son arrojados a la costa junto al templo de Venus en donde se refugian la joven y su esclava; ésta, cuando va a buscar agua se encuentra a Tracalión, criado de Pleusidipo, y le cuenta cómo naufragaron y perdieron en el mar los «objetos de reconocimiento» de Palestra que el lenón puso en una bolsa; Lábrax reclama a las mujeres en el templo, Démones consigue esconderlas en su casa, mientras un pescador, Gripo, ha pescado la bolsa que contiene el cofrecillo con los objetos de reconocimiento, Tracalión lo ve con ella y después de una disputa sobre la propiedad de ésta, en la que Démones será el árbitro, finalmente, los objetos llegarán a manos de Palestra quien los nombrará, uno a uno, frente a su padre, entonces éste la reconocerá: final feliz, como quiere y espera el público de la Comedia 9 .

En el Acto IV, escena II, aparece por vez primera Gripo, pescador esclavo de Démones, que, según sabremos por el contenido de su monólogo, lleva una red que envuelve una bolsa de la que pende una cuerda ${ }^{10}$ que lleva arrastrando.

Este personaje pronuncia un monólogo en el que alaba su propia diligencia y cuenta cómo ésta le fue recompensada con una pesca muy particular. En un momento determinado comienza a hacerse esperanzas acerca de lo que ha pescado (vv. 924-926):

Nam ego nunc mihi, qui impiger fui,

Repperi ut piger si uelim siem.

${ }^{7}$ En este tipo de argumentos, los raptores de inocentes muchachas, generalmente piratas o bandidos, las venden sistemáticamente como esclavas, y, aveces, a crueles dueños de burdeles; así sucede por ejemplo en la novela Historia Apollonii regis Tyri.

* Se ajusta así a la imagen de personaje despreciable que el público romano (no así el griego) espera que ofrezca todo lenón.

9 "...termina de modo contrario para los buenos y para los malos. Y parece ser la primera por la flojedad del público; pues los poetas al componer se pliegan al deseo de los espectadores, pero este no es placer que debe esperarse de la tragedia sino que más bien es propio de la comedia; aquí, en efecto, hasta los más enemigos... al fin se toman amigos...» (Aristóteles, Poética 1435a). Empleo para la traducción la edición trilingüe de García Yebra, Madrid 1974.

10 Esta cuerda es la que da el título a la obra, Rudens. El término aparece por vez primera en la escena III del Acto IV, cuando Tracalión, que ha visto a Gripo con la bolsa, lo detiene y le pide que se pare: Dum hanc tibi quam trahis rudentem complico, «Mientras te enrollo esta cuerda que arrastras" (v. 938). 
Hoc ego in mari quicquid hic inest

Repperi; quicquid inest graue quidemst; aurum hic

Ego inesse reor; Nec mihi conscius est ullus homo. (vv. 924-926)

Pues ahora yo que no fui perezoso, he encontrado la forma de ser perezoso, si quisiera. Esto, sea lo que sea lo que haya aquí dentro, lo encontré en el mar; sea lo que sea lo que haya dentro, es realmente pesado. Yo creo que aquí dentro hay oro. Y ningún hombre lo sabe mas que yo. ${ }^{11}$

Y, animándose con sus propias palabras, comienza a hacer las «cuentas de la lechera» (vv. 927-939):

Tunc haec tibi ocassio, Gripe, optigit, ut liberet ex tempulo praetor te

Nunc si faciam, sic consilium est: ad erum ueniam docte atque astu,

Pauxillatim pollicitabor pro capite argentum, ut sim liber.

lam ubi liber ero, igitur demum instruam agrum atque aedis, mancipia;

Nauibus magnis mercaturam faciam; apud reges rex perhibebor. Post animi causa mihi nauem faciam atque imitabor Stratonicum.

Oppida circumuectabor

Ubi nobilitas mea erit clara

Oppidum magnum communibo:

Ei ego urbi Gripo indam nomen, Monimentum meae famae et factis,

bi qui regnum magnum instituam.

Magnam res hic agito in mentem

Ahora, Gripo, se te presenta esta ocasion para que inmediatamente el pretor te dé la libertad. Ahora actuaré así, así es mi plan: iré ante el amo con habilidad y astucia, poquito a poquito le propondré un rescate, para ser libre. Cuando ya sea libre entonces por fin compraré un campo y una casa, y esclavos. Me dedicaré al comercio con grandes naves, me presentaré entre los reyes como un rey, después, por gusto, me haré una nave e imitaré a Estratónico ${ }^{12}$, recorreré sucesiva-

11 Traduzco a partir de la edición de Ernout citada en nota 5.

12 Parece que se trataba de un citarista famoso en la época de Alejandro que se dedicaba a recorrer las más importantes ciudades de Grecia y que logró gran fama. Sin embargo las fechas son muy discutidas, lo que sí parece evidente es que cuando Difilo - autor griego en quien se basa la obra de Plauto- escribió su comedia el recuerdo de este personaje aún debía de estar vivo. Su 
mente las ciudades, cuando sea un personaje famoso, haré construir una gran ciudad amurallada, a esta ciudad le daré el nombre de Gripo, en recuerdo de mi fama y hazañas, pues allí fundaré un gran reino.

Hasta aquí los «castillos en el aire» ${ }^{13}$, claramente desmesurados ${ }^{14}$. Después la paulatina vuelta a la realidad: Magnam res hic agito in mentem, «Aquí en mi mente proyecto hacer grandes cosas». Ahora hay que ser práctico: Hunc nunc uidulum ${ }^{15}$ condam, «De momento voy a esconder esta bolsa», y realista: Sed hic rex cum aceto pransurust/ et sale, sine bono pulmento, «Pero este rey va a comer con vinagre y sal, sin un buen guiso.»

Los «sueños» de este esclavo como los de todos los protagonistas de la fábula de «la lechera» se irán al traste: no le dará tiempo a esconder la bolsa, Tracalión lo verá con ella, discutirán en un sabroso y divertido enfrentamiento y, finalmente los objetos de la bolsa y el dinero volverán a sus auténticos dueños. Más adelante volveremos al desarrollo del cuento. Ahora veamos si hay restos del tema en el mundo antiguo.

Es de sobra sabido que los poetas cómicos latinos fueron ante todo, traductores y adaptadores de la Comedia Nueva griega, aunque ello no significa que carecieran de vena creadora e hicieran gala de ella; lo que hacen los comediógrafos latinos es reescribir las obras recibidas de los griegos, darles su toque romano, adaptarlas al gusto de su público, eso sí, Plauto y Terencio cada uno en su estilo.

El propio Plauto nos revela que el modelo para Rudens fue una obra original del poeta cómico griego Dífilo ${ }^{16}$, poniendo en boca del narrador del Pró-

presencia en la obra de Plauto ratifica la existencia de un original griego, pero seguramente sus espectadores romanos no entenderían la alusión. W. S. Anderson (1980) («Gripus and Stratonicus. Plautus, Rudens 930-936», American Journal of Philology, CVII, 560-563) hace una sugerencia muy atractiva respecto a lo que llama «creative mistake» en Plauto.

1. Sueños fantásticos que otro ex-exclavo consigue alcanzar: Trimalción en el Satyricon de Petronio. Como Gripo en sueños, el nuevo rico consiguí la libertad, casa, esclavos, campos y recorrió los mares dedicándose al comercio en grandes naves, porque asegura: magna nauis, magnam fortitudinem habet (76). Claro que a Trimalción hay que creele sólo a medias, ya sabemos cómo es. Sea como sea, ya señala W. S. Anderson (1980:561) «Dream of riches in connection with trading overseas form a common theme of New Comedy and later Literature». Recordemos, sin ir más lejos la comedia Mercator del mismo Plauto en la que un padre para que su hijo olvide a una cortesana, lo envía a ejercer el comercio por los mares como antes también lo hizo su padre. En la novela Historia Apollonii regis Tyri, el protagonista también se embarca para dedicarse al comercio después de la muerte de su esposa.

${ }_{14}$ Como luego sabremos, dentro de la bolsa había dinero suficiente para conseguir alguno de estos deseos.

13 Uidulum bolsa de cuero para viaje.

16 Se han sugerido los siguientes títulos: «La bolsa de viaje», «La bolsa», «El Arbitraje»; también se ha sugerido «La balsa» modelo de la Vidularia (F. della Corte). Y es que esta comedia 
logo, el Astro Arcturo, cuando narra el argumento de la comedia y sitúa la acción, las siguientes palabras: Primundum huic esse nomen urbi Diphilus/ $C y$ renas uoluit, «En primer lugar, Dífilo quiso que el nombre de esta ciudad fuese Cirene».

No es posible localizar lo que en la obra pertenece a Dífilo y lo que es totalmente de Plauto ${ }^{17}$, por ello nunca podremos saber si este "cuento de Gripo» estaba ya en la comedia griega original. Resulta sin embargo a este respecto muy sugerente lo que señala $S$. Trenkner ${ }^{18}$. Según esta estudiosa el tema era ya conocido en la antigüedad, y aduce como prueba en primer lugar, dos fragmentos de Epicarmo ${ }^{19}$ pertenecientes al drama ${ }^{20}$ titulado Las ollas. La traducción de los textos es como sigue ${ }^{21}$ :

...pero sin embargo, unas hermosas y gruesas corderas me producirán diez monedas con su venta, pues son de una madre tal... 207 (136 K.).

Yendo como licitador, cómprame inmediatamente una hermosa ternera de diez monedas... 208 (137 K).

Fue Crusius ${ }^{22}$ quien propuso que en estos fragmentos nos encontraríamos ante una versión de la fábula de la lechera en la que un vendedor de ollas haría «castillos en el aire» sobre sus futuras riquezas ${ }^{23}$.

presenta interesantes e importantes similitudes con Rudens; la trama resulta parecida, pues como Rudens parece tratarse de una comedia de reconocimiento: hijo raptado que se convierte en esclavo de su padre, naufragio, pérdida de baúl con objetos de reconocimiento, pescador que la rescata. disputa por la bolsa, arbitraje de padre,... La idea de que Plauto hubiera reelaborado dos veces el mismo original, aunque menos verosímil, no se descarta.

17 Señala ERnOUT, A., (1962: 111) que el erudito alemán MARX, F., intentó una reconstrucción en su edición comentada de la comedia (Leipzig 1928).

${ }^{18}$ En su documentada y utilísima obra The Greek Novella in the Classical Period. Cambridge $1958,126$.

${ }^{19}$ Comediografo griego a quien se suele situar entre el 528-428 a.d. C, y al que los antiguos atribuyen la invención de la comedia, ya que las suyas son las obras cómicas más antiguas de las que se tenía y tiene constancia. «Quien introdujo máscaras o prólogos o pluralidad de actores se desconoce; pero el componer fábulas, Epicarmo y Fotis» (Aristótes, Poética 1449b 5-6)

${ }^{20}$ Los griegos llamaban a las obras de Epicarmo drámata, distinguiéndolas de las comedias áticas posteriores de características diferentes.

${ }_{21}$ Empleo la edición de RODRIGUEZ-NORIEGA, L. (1996) Epicarmo de Siracusa. Testimonios y fragmentos, edición crítica bilingüe, Oviedo, 131-132. Se trata esta de una de esas obras de Epicarmo «cuyo argumento versa en torno a personajes y situaciones de la vida cotidiana, con evidente vinculación con el mimo...» (RODRIGUEZ.-NORIEGA, XV)

22 «Epicharm bei Paremiographen», Philologus, Suppl. VI 1892, 293-294.

23. RODRIGUEZ-NORIEGA (1996: 131); se indican también aquí otras interpretaciones diferentes. 
El otro texto que propone Trenkner es precisamente el que nos ocupa. Regresemos pues a la versión que nos ofrece Plauto, totalmente original, y detengámonos brevemente en algunos aspectos de ella: combinación de motivos, adaptación del cuento a la escena y su función dentro de la obra.

La originalidad de la versión viene determinada en primer lugar por el marco en el que se inserta, por el contexto que la rodea; en este caso no estamos ante una recopilación de cuentos dentro de una historia marco, o diálogo entre el maestro y el discípulo, o bien una colección de fábulas en las que unas y otras constituyen un contexto homogéneo, en nuestro caso la fábula de la lechera se inserta en la trama de una historia principal, de forma tal que se convierte a la vez en un relato secundario y un acontecimiento determinante en el desarrollo de dicha historia principal. Ésta, que ya resumimos más arriba cuando contábamos el argumento de la comedia, está trenzada mediante un conjunto de variados motivos ${ }^{24}$; pues bien, es precisamente la presencia y el desarrollo de estos motivos el que influye en la versión de la fábula. Así, los motivos de la «tempestad» y el «naufragio», suele tener como contrapartida la aparición del motivo del «pescador»; este suele desempeñar el papel de «ayudante» ${ }^{25}$ acogiendo y alimentando a los personajes que después del naufragio llegan a la orilla arrojados por el mar. En Rudens se trata de Gripo y su ayuda no será aquella que estamos habituados a encontrar sino que vendrá determinada por un motivo central de la historia principal: el «reconocimiento».

${ }^{24}$ Thompsom (1972:528) describe el motivo como el elemento más pequeño del cuento que tiene el poder de perdurar en la tradición. Para tener este poder ha de tener algo poco usual y perdurable. Muy claras y sugerentes, como siempre en este autor, son las páginas de KAYSER, W., (1985) Interpretación y análisis de la obra literaria, Madrid, 75-98, dedicadas al Motivo: «...la observación ha demostrado que cuanto mejor se estudian las leyendas y cuentos de los diversos pueblos, más semejanzas se descubren, no sólo en pequeños rasgos comunes, sino también en las situaciones, en los personajes, en los esquemas. Trátanse... de unidades que aparecen en las más diversas combinaciones... Estas unidades se designan con el nombre de motivos. Dondequiera que los encontremos, en una leyenda o en cualquier obra literaria, siempre se presentarán de manera concreta» (76). También Segre, C., (1985) Principios de análisis del texto literario, Barcelona, 359 , sobre la distinción tema/motivo: «Llamaremos temas a aquellos elementos estereotipados que sostienen un texto o gran parte de él; los motivos son, por el contrario, elementos menores y pueden estar presentes incluso en un número elevado. Muchas veces el tema resulta de la insistencia de muchos motivos. Los motivos tienen mayor facilidad para manifestarse en el plano del discurso linguístico, tanta que, si se repiten, pueden actuar de forma parecida a los estribillos. Los temas son generalmente de carácter metadiscursivo. Los motivos constituyen, habitualmente resonancias discursivas de la metadiscursividad del tema».

25. Por ejemplo en Vidularia (cf. n. 16); también en la novela griega: Jenofonte de Éfeso, Efesíacas V, 1; Heliodoro, Teágenes y Cariclea V.18; Historia Apollonii regis Tyri, 12. Incluso en el Satyricon (115) de Petronio, después de una tormenta con naufragio incluido, aparecen unos pescadores, bastante miserables, eso sí, pero luego los náufragos pasan la noche en la cabaña de un pescador. 
En casi todas las historias de separaciones familiares muy largas, hay un momento en el que asistimos al reencuentro, al reconocimiento. La comedia Rudens, no es una excepción y de hecho, toda la peripecia se centra en la separación padre-hija y en las situaciones que van a propiciar el reconocimiento. Sabemos por Aristóteles ${ }^{26}$ que el reconocimiento suele producirse mediante recursos diversos; en este caso, aunque no es el más apreciado por el sabio griego $^{27}$, el reconocimiento se producirá mediante objetos ${ }^{28}$.

Por tanto, la ayuda del pescador consistirá en el rescate del fondo del mar de los perdidos «objetos de identificación» de la protagonista facilitando el desenlace.

Pero hay en este rescate, en esta pesca fortuita y en la interpretación que Gripo hace de ella, una contaminación con otro motivo que ya no pertenece a la historia principal sino a la historia secundaria del propio Gripo: "el tesoro encontrado»; éste es precisamente el desencadenante de nuestra versión de la fábula de la lechera, de «las cuentas de la lechera» del pobre pescador.

Asegura THOMPSOM ${ }^{29}$ que los cuentos sobre búsquedas de tesoros han sido comunes a todas las civilizaciones. «El tesoro encontrado» está en el folklore relacionado con «lo maravilloso», cosas imaginarias comunes a toda la humanidad. Según Thompsom (1972: 345):

En una vida llena de luchas por la alimentación y el abrigo, ...no es de dudar que una criatura como el hombre imagina las condiciones bajo las cuales desaparezcan todas sus privaciones... En algunos ánimos estas nociones son quiméricas y fuerzan una realización de la vida tal como se vive actualmente, vida en la que uno solo necesita grandes tesoros o dinero para comprar todo lo que desea... ¿Por qué no podría cualquiera, si es lo suficientemente sagaz, descubrir tales tesoros y vivir como un rey?

La Comedia latina, se desenvuelve en un mundo real, y Gripo, el pescador vive en ese mundo real de miseria, como lo confirma el monólogo de los

${ }^{26}$ Cf. Poética 1452a-1452b.

27 4...y el reconocimiento más hermoso es el que se produce junto con peripecia...En cuanto a las clases de reconocimiento, en primer lugar hay la que está más alejada del arte por encontrarse en situación apurada: es la que se produce por signos externos....Pero el reconocimiento más apropiado a la fábula y el más apropiado a la acción es el mencionado...

${ }^{28}$ Estos dos motivos son muy frecuentes y conocidos en la literatura antigua desde Heródoto (I, $108 \mathrm{ss}$.) hasta la novela griega: se citan objetos de reconocimiento en Dafnis y Cloe de Longo, y Teagenes y Cariclea de Heliodoro. Siempre ambos están asociados a temas de separaciones farmiliares.

${ }^{29}$ ThOMPSOM, S., (1972) El cuento folklórico, Caracas, 345 ss. 
pescadores que abre el Acto II, Escena I del Rudens, que es casi una justificación de las quimeras de Gripo (vv. 290 ss.):

Los hombres que son pobres viven de todos modos hechos unos miserables... Por nuestros ropajes ya podéis saber muy bien lo ricos que somos nosotros. Estos anzuelos y estas cañas de pescar son para nosotros ganancia y sustento. Diariamente vamos a la mar en busca de alimento...

El hecho de encontrar un tesoro no es ajeno a la comedia - así ocurre en Aulularia $-{ }^{30}$, y al cuento folklórico; pero casi siempre, como dice THOMPSOM (1972:345) «Aún después de encontrado el tesoro rara vez se consigue la alegría esperada». Y así ocurre en el caso de nuestro pescador; el propio Plauto delata la contaminación de motivos cuando hace que Gripo, enfadado porque ve que va a perder su tesoro maldiga airado (vv.1256-1257):

At ego deos quaeso, ut quidquid in illo vidulost

Si aurum, si argentum est, omne id ut fiat cinis

Y yo pido a los dioses que lo que sea que haya en la bolsa, ya sea oro o plata, se convierta por completo en cenizas

Gripo se está comportando como un experto en folklore en su maldición: el lado «maravilloso» del tesoro tiene también su lado oscuro; Thompsom cuando trata de este motivo destaca la variante en la que el tesoro, una vez que uno se ha enriquecido y cree que está a salvo, desaparece y se convierte en carbón o virutas ( $\mathrm{N} 558$ ).

La historia secundaria de Gripo, su cuento, comenzaría con el encuentro del tesoro y se prolonga, hasta que se produce el desenlace (consigue la libertad), con una serie de pasos en los que el pescador, en estos momentos protagonista, se resiste a la entrega de su «tesoro»: enfrentamiento y disputa con Tracalión respecto a quien es el dueño de la bolsa, arbitraje sobre el asunto por parte de su amo Démones ${ }^{31}$, y engaño del lenón; en estos pasos el pescador ve cómo su «cántaro» se va haciendo pedazos.

30 Es interesante resaltar que los fragmentos de Epicarmo a los que nos referimos al principio del estudio y que se piensa pertenecen a la Comedia Las ollas, se adscribieron en un principio a una comedia a la que se dio el título de El tesoro, que trataría de un hombre que encuentra un tesoro en una olla, como en Aulularia.

${ }^{31}$ Este tipo de disputa y arbitraje también se dan en Epitrepontes de Menandro y Alope de Eurípides (Hyg. fab. 187). 
La historia secundaria de Gripo y la historia la principal convergen cuando Tracalión ve y detiene al pescador. Su papel de «oponente» dentro del relato principal tiene la función de dilatar el desenlace, de hacer la espera más interesante y divertida.

La originalidad de la versión también reside en su adaptación a la escena: en casi todas las versiones conocidas de la fábula, un narrador omnisciente se sitúa detrás del personaje y nos hace conocer su pensamiento que se presenta en estilo directo, en forma de monólogo interno; después, el narrador retoma la palabra para contarnos el desenlace, es decir, la forma en que se pierde el objeto sobre el que se fantasea, y la moraleja. Todas las versiones que conocemos se presentan bajo la forma de relatos ${ }^{32}$. Sin embargo la fábula de Gripo está trasladada a la escena y en la escena no hay narrador. Como dice Segre ${ }^{33}$, en la obra teatral se elimina la mediación del Yo narrador o personaje narrador. El texto está constituido sustancialmente por los enunciados de varios Yo personaje que pueden incluir, en forma diegética, la narración de los hechos externos a la escena.

En la versión de Plauto, Gripo suple la función del narrador apareciendo en escena y arrastrando la bolsa, luego dice lo que ha hecho, está haciendo y, sobre todo piensa hacer, en un monólogo. Con él se produce un acercamiento entre personaje y público, mientras la relación entre éste y el autor queda oculta ${ }^{34}$. Este acercamiento sin duda confiere viveza a la fábula y consigue que el personaje que va a perder lo que tiene resulte más real, más próximo ${ }^{35}$.

El monólogo desempeña en Rudens, creemos, una doble función ${ }^{36}$, la de caracterizar a un personaje nuevo que acaba de aparecer por vez primera en escena -uso frecuente en Plauto- y la de plantear dos perspectivas distintas en el desarrollo de la trama: por un lado es el anuncio de una solución del conflicto (los «objetos de identificación» han sido rescatados) y a la vez anuncio de un obstáculo para ello (el pescador se va a resistir a soltar la presa), Gripo, como ya hemos visto, se ha convertido a la vez en "ayudante» y «oponente».

32 El profesor Fradejas, en su artículo citado en n.4, presenta una versión de la fábula en la escena que aparece en Historia de una escalera de BUERO VALLEJO ANTONIO.

33 SEGRE, C. (1984) Teatro e Romanzo, Turín, 16

34 SEgre (1984: 7)

35 Como señala Slater en su interesante estudio Plautus in Performance, Princeton-New Jersey, 1985, 75, citando a STYAN, S.L.: «The fundamental purpose of the aside or soliloquy is to engage the spectator directly, to throw him a face-to-face challenge to agree or desagree: they are a reminder to all that a play is in progress".

36 Para el valor de los monólogos en Plauto cf. SLATER (1985), y también Frenkel E. (1960) Elementi plautini in Plauto, Florencia. También es interesante, PrESCOTT, H.W. (1939) «Link monologues in Roman Comedy», CP 34, 1-23, 116-126. 
Dentro de la Comedia, el personaje se autocaracteriza mediante el monólogo, pero detrás de esa confesión, se vislumbra sin duda la figura del autor, quien nos quiere dar, usando al propio personaje, una idea muy precisa de él; el autor hace del personaje lo que quiere, y lo que quiere Plauto es mostrarnos la figura de un pobre hombre que sueña despierto cosas imposibles; se trata en realidad de una versión del «bobo» que Plauto dibuja, sin lugar a dudas mediante la desmesura de los sueños de Gripo, y que resalta con un guiño al espectador dentro del monólogo de su personaje cuando le hace afirmar: «Yo creo que aquí dentro hay oro. Y ningún hombre lo sabe más que yo». El autor, en cierta forma, se está riendo de él de cara a la audiencia, porque el público, que ya sabe más que el personaje ve cómo éste se está equivocando, pues en el Acto Il el autor se ha ocupado de que Tracalión, tras escuchar el relato de $\mathrm{Pa}$ lestra en el que cuenta que su cofrecillo se ha hundido en el mar, comente para sí mismo como en una premonición ${ }^{37}$ : «Tengo la intuición de que alguien se ha sumergido y la ha sacado".

Esta figura del «bobo» también aparece asociada a la fábula en el folktale. Thompson ${ }^{38}$ sitúa el motivo de «castillos en el aire» con sus diferentes versiones dentro de lo que denomina cuentos simples, concretamente de «tontos y bobos".

Pero el personaje de la Comedia es más complejo que el del cuento, naturalmente, y los rasgos del «bobo» en Plauto se afinan hasta que lo convierten en un aprovechado que se cree muy listo, pero es burlado finalmente por la honradez de Démones y la desvergüenza del lenón, todo ello mediante situaciones e intervenciones de Gripo que causan la hilaridad del público.

En el desarrollo de la fábula la cercanía del personaje conseguida con el monólogo se mantiene, ya que es el mismo Gripo quien confiesa cuál fue su error, su «patada al cántaro».

En la primera ocasión, maldiciendo a Tracalión y a sí mismo de esta manera (vv. 1166-1169):

Qui te di omnes perdant, qui me hodie oculis uidisti tuis

Meque adeo scelestum, qui no circumspexi centiens

Prius me nequis inspectaret, quam rete extraxi ex aqua

${ }^{37}$ Se trata de una prolepsis o anticipación interna. Estas anacronías son mucho menos frecuentes que las retrospecciones, «se reducen en su mayoria a una simple alusión...al desenlace de la fábula, un desenlace que se debe conocer para reconocer (retrospectivamente) las anticipaciones como tales», cf. GENETTE, G., (1972) Figures III, París, 105, y BAL., M. (1987) Teoría de la narrativa. Una introducción a la narratología, Madrid, 71.

${ }^{38}$ Thompsom, S., (1972: 256 ss.) 
¿Que todos los dioses te maldigan, a ti que me has visto con tus ojos, y maldito también yo, por no haber mirado alrededor cien veces a ver si alguien me espiaba, antes de sacar la red del agua! ${ }^{39}$

La segunda vez, de nuevo en un monólogo, cuando ya le han quitado la bolsa (vv. 1184-1185):

Sumne ego scelestus, qui illinc hodie excepi uidulum?

Aut cum excepi, qui non alicubi in solo abstrusi loco?

¿No soy un desgraciado por haber sacado hoy esta bolsa?

¿O, al menos, cuando la saqué, por no haberla escondido en algún sitio, en un lugar solitario?

Aquí el autor es más benévolo con su personaje quien se ve a sí mismo como falto de precaución para conservar lo conseguido; de esta forma, en la tradición de esta fábula, se ajustaría a la clasificación del Panchatantra que la sitúa en la serie denominada «La conducta impremeditada».

A través de lo hasta ahora expuesto hemos visto cómo la fábula desempeña una función esencial en la obra proporcionando escenas cómicas (enfrentamientos ${ }^{40}$, juegos de palabras, etc...), contribuyendo así a una de las finalidades del género: divertir. También se ha puesto de manifiesto la función clave que desempeña dentro de la historia propiciando su desenlace, y esto es evidente hasta tal punto que es el mismo Plauto quien de nuevo nos facilita el análisis; esta vez es en el argumentum de la obra en donde nos hace el guiño con su selección de acontecimientos y su forma de situarlos; juzguen si no el texto:

\section{Argumentum}

Reti piscator de mari extraxi uidulum

Ubi erant erilis filiae crepundia.

Dominum ad lenonem quae subrepta uenerat.

Ea in clientelam suipte inprudens patris

Naufragio eiecta deuenit. Cognoscitur

Suoque amico Pleusidippo iungitur.

${ }^{39}$ En uno de los fragmentos de la Vidularia ( $c f$. n.16) se conservan estas palabras del pescador Cacisto: « $¡$ No hay hombre más desdichado y desgraciado que yo! ¡Decir que, cuando vi el baúl, no se me ocurrió mirar cien veces en torno mío!

40 El duelo verbal típico de un juicio a cerca de la propiedad de la bolsa que mantienen Gripo y Tracalión, tuvo que resultar sumamente divertido para los romanos tan amantes del género cuando también lo es para nosotros. 
Un pescador extrajo del mar una bolsa, en donde estaban unos juguetes de la hija de su amo que tras haber sido raptada, había caído en poder de un lenón. Ella, salvada de un naufragio, sin saberlo, recurrió a la protección de su propio padre. Fue reconocida y se casó con su amante Pleusidipo.

La fábula de la lechera, como todos sabemos desde niños, siempre termina mal, sin embargo, en la versión que nos ofrece en Rudens, una de sus comedias más románticas y amables, Plauto, al final de la obra, se permite un gesto de ternura hacia su tosco pero soñador personaje, y por medio de Démones su amo, gracias al dinero del infame lenón, le concede el que fue el primer peldaño ${ }^{41}$ de su sueño: la libertad.

${ }^{41} \mathrm{Y}$ uno de los pocos que podría haber conseguido realmente si su destino de perdedor no hubiera evitado que se quedase con el contenido de la bolsa. 